\title{
Lagrangian simulation and analysis of the power-law fluid mixing in the two-blade circular mixers using a modified WCSPH method
}

\author{
Rahim Shamsoddini ${ }^{1}$, Mohammad Sefid ${ }^{2}$ \\ ${ }^{1}$ Sirjan University of Technology, Department of mechanical engineering, Sirjan, Iran, Postal code: 7813733385 \\ ${ }^{2}$ Yazd University, School of Mechanical Engineering, Yazd, Postal Code: 89195-741 \\ "Corresponding author: e-mail: shamsoddini@stu.yazd.ac.ir
}

\begin{abstract}
In the present study, we introduce a robust modified Weakly Compressible Smoothed Particle Hydrodynamics (WCSPH) method in order to examine miscible mixing within a two-blade paddle mixer. Since it has a Lagrangian nature and it is based on particles, Smoothed Particle Hydrodynamics (SPH) is an appropriate and convenient method for simulating the moving boundary problems and tracking the particles in the mixing process. The present study thus introduces a convenient SPH method for modelling the mixing process for the power-law fluids. Two geometries for the mixer are examined and the effects of the power-law index on the fluid mixing are investigated. The results show that the geometric change from circular chamber to twin chamber considerably increases the mixing rate (by at least 49\%). The results also indicate that the twin chamber mixer is more efficient for the fluids with higher power-law index.
\end{abstract}

Keywords: two-blade mixer, twin chamber, Lagrangian, power-law.

\section{INTRODUCTION}

Mixing, as a transport phenomenon, is usually carried out to reduce inhomogeneity for species, temperatures, and phases. Fluid mixing is one of the most important phenomena in the chemical and medical industries. It is a crucial process in many of the devices used in the biochemical process, production of nucleic acids, drug delivery, and polymer industries.

Two different types of mixers, stirred tanks and static mixers, are commonly used in extensive range of industrial applications ${ }^{1}$. In the present study, a paddle mixer that includes two blades in order to improve mixing is investigated. The two well-known approaches traditionally used for simulating mixing flow are the Eulerian-Eulerian and Eulerian-Lagrangian methods. The Eulerian-Eulerian method assumes the secondary phase to be a continuum phase and applies the mass conservation equation in order to detect the concentration. By contrast, the Eulerian-Lagrangian method applies the dynamics of a single particle by using the trajectory method $^{2}$. Hence, this approach assumes a continuum media for the liquid phase and traces the discrete phases using Lagrangian particle trajectory methods ${ }^{3}$. The accuracy of the Eulerian-Eulerian method depends on the empirical constitutive equation, which is used. Further, this approach has restrictions in predicting certain discrete flow characteristics. However, the disadvantage of the Eulerian-Lagrangian method is its requirement for more extensive computing time ${ }^{3}$.

Recently, full Lagrangian approaches have been used to model the mixing phenomena ${ }^{4-6}$; these methods are based on the particle methods. Among the particles methods, Smoothed Particle Hydrodynamics (SPH) is one of the first approaches used to simulate mixing phenomena; Robinson et al. ${ }^{5}$ modelled a twin cam mixer using SPH, Lenaerts and Dutré ${ }^{4}$ simulated both fluid and granular material using a unified SPH framework, and Orthmann and $\mathrm{Kolb}^{6}$ proposed a fast and consistent SPH model which was suitable for convection-diffusion models of incompressible fluids, applying their temporal blending scheme to reduce the number of particles in the simulation.

No modelling is needed for convective terms for the SPH modelling; convective terms are satisfied directly by the motion of the particles. Each particle in the mixing flow can also be directly tracked. The SPH method was first proposed for astrophysical applications by Gingold and Monaghan ${ }^{7}$ and Lucy ${ }^{8}$. Since then, it has been extended to model a wide range of engineering applications such as fluid flows and transport phenomena. For SPH, two approaches are generally used to specify the pressure field. The first of these, known as Weakly Compressible SPH (WCSPH), uses an appropriate equation of state to relate pressure variations to density variations. The second, incompressible SPH (ISPH), solves the Poisson pressure equation in order to detect the pressure. The SPH is also a proper method for moving boundary and fluid-structure interaction (FSI) problems because of its Lagrangian nature, and thus has widely been used to simulate FSI problems ${ }^{9-12}$.

In the present study, a robust modified WCSPH is introduced and used to simulate the mixing phenomena in a two-blade paddle mixer. An ISPH method is also available, which has been examined for the mixing phenomena ${ }^{13}$. However, WCSPH is a fully explicit method which is implemented more easily than ISPH, although the former suffers from non-physical fluctuations. In the present study, a new algorithm is introduced to reduce the non-physical fluctuations of the WCSPH method. As such, a proper method is applied to simulate two-blade paddle mixers. Paddle mixers are mixing devices consisting of one or more blades mounted on rotating shafts. The main advantages of paddle mixers are their simplicity and $\operatorname{cost}^{14}$. They are used in the micro and macro-scales. The paddle mixers have widely been investigated by using the experimental and numerical methods ${ }^{15-19}$. However, the numerical methods used are usually Eulerian methods. In this study, a fully Lagrangian approach is introduced for simulation of the two-blade mixers. In addition, it is assumed that the fluids are non-Newtonian power-law and miscible fluids. Although the advection is the main 
part of the mixing process in the paddle mixers, the molecular diffusion is also an important part of mixing for the miscible fluid, especially in the laminar flows. So, the diffusion equation is also considered and solved.

Reynolds number is assumed to be low $(\operatorname{Re}<10)$. High viscosity liquids mixing or micro scale mixing are examples of low Reynolds fluids mixing. High viscosity mixers are mixers designed for laminar mixing processes. Some products that may need laminar mixing in a high viscosity mixer include putties, chewing gum, and soaps ${ }^{\mathbf{2 0}}$.

In the following, the formulation and numerical procedure are first discussed and then the results and discussion are presented.

\section{NUMERICAL PROCEDURE}

\section{SPH formulation and governing equations}

The SPH formulation is based on an integral form which indicates that each continuous defined function $f$ over an interest domain $\Omega$ can be stated as:

$f(\boldsymbol{r})=\int_{\Omega} f(\boldsymbol{r}) W\left(\boldsymbol{r}-\boldsymbol{r}^{\prime}, h\right) d \boldsymbol{r}^{\prime}$

where $r$ and $r$ ' are respectively position vector and sub integral variable, $W$ is the weight or kernel function, and $h$ is the smoothing length. This equation is approximated by a numerical summation on the discrete points in the domain $\Omega$ :

$f(\boldsymbol{r})=\sum_{j} \forall_{j} f_{j} W\left(\boldsymbol{r}-\boldsymbol{r}_{j}, h\right)$

where $\forall$ is the volume of the $j$-th particle. In the present study, the fifth-order Wendland kernel is used ${ }^{21}$. Dehnen and $\mathrm{Aly}^{22}$ showed that the use of this kernel function improves the accuracy in the fluid flow modelling.

$W(\boldsymbol{r}, h)=W_{0} \times\left\{\begin{array}{lc}\left(1-\frac{|\boldsymbol{r}|}{h}\right)^{4}\left(4 \frac{|\boldsymbol{r}|}{h}+1\right) & 0 \leq \frac{|\boldsymbol{r}|}{h}<1 \\ 0 & \frac{|r|}{h} \geq 1\end{array}\right.$

where $W_{0}$ is $7 / \pi h^{2}$ for two-dimensional cases.

The gradient, divergence, and Laplacian operator for an arbitrary scalar function $f$ or tensor function $\boldsymbol{F}$ are respectively:

$$
\begin{aligned}
& \langle\nabla f\rangle_{i}=\sum_{j} \forall_{j}\left(f_{j}-f_{i}\right) \boldsymbol{B}_{i} \cdot \nabla W_{i j} \\
& \langle\nabla \cdot \boldsymbol{F}\rangle_{i}=\sum_{j} \forall_{j}\left(\boldsymbol{F}_{j}-\boldsymbol{F}_{i}\right) \cdot\left(\boldsymbol{B}_{i} \cdot \nabla W_{i j}\right) \\
& \left\langle\nabla^{2} f\right\rangle_{i}=\widehat{\boldsymbol{B}}_{i}: \sum_{j} 2 \forall_{j} \boldsymbol{e}_{i j} \nabla W_{i j}\left(\frac{f_{j}-f_{i}}{r_{i j}}-\boldsymbol{e}_{i j} \cdot\langle\nabla f\rangle_{i}\right)
\end{aligned}
$$

where $\boldsymbol{e}_{\mathrm{ij}}$ is the unit vector in the inter-particle direction (from $j$ to $i$ ), $\boldsymbol{B}$ is a corrective tensor for kernel gradients which was previously applied and examined by Bonet and $\mathrm{Lok}^{23}$ :

$$
\boldsymbol{B}_{i}=-\left[\sum_{j} \forall_{j} \boldsymbol{r}_{i j} \nabla W_{i j}\right]^{-1}
$$

and $\widehat{\boldsymbol{B}}_{\boldsymbol{i}}$ is a renormalization tensor offered by Fatehi and Manzari ${ }^{24}$, which is calculated using the following set of equations:

$\widehat{\boldsymbol{B}}_{i}:\left[\sum_{j} \forall_{j} \boldsymbol{r}_{i j} \boldsymbol{e}_{i j} \boldsymbol{e}_{i j} \nabla W_{i j}+\left(\sum_{j} \forall_{j} \boldsymbol{e}_{i j} \boldsymbol{e}_{i j} \nabla W_{i j}\right) \cdot \boldsymbol{B}_{i} \cdot\left(\sum_{j} \forall_{j} \boldsymbol{r}_{i j} \boldsymbol{r}_{i j} \nabla W_{i j}\right)\right]=-\boldsymbol{I}$
Eq. (6) is introduced and applied by Fatehi and Manzari $^{24}$. In the present study, Eq. (6) is used for the diffusion terms in the transport equations.

The governing equations are respectively mass, momentum, pressure state, and concentration transport equations:

$\frac{d \rho}{d t}=-\rho \nabla \cdot V$

$\rho \frac{d \boldsymbol{V}}{d t}=\nabla \cdot\left(\mu_{p}\left(\nabla \boldsymbol{V}+\nabla \boldsymbol{V}^{T}\right)\right)+\rho \boldsymbol{g}-\nabla p$

$p-p_{0}=c^{2}\left(\rho-\rho_{0}\right)$

$\frac{d C}{d t}=\alpha \nabla^{2} C$

where $\rho, V, p, \alpha, C$, and $c$ are respectively the fluid's density, velocity, pressure, mass diffusivity, concentration, and speed of sound. $\mu_{p}$ is apparent viscosity, which is given by:

$\mu_{p}=k \gamma^{n_{p}-1}$

where $k$ is consistency index, $n_{p}$ is power-law index, and $\dot{\gamma}$ is

$\dot{\gamma}=\sqrt{\frac{1}{2} \sum_{\alpha} \sum_{\beta}\left(\frac{\partial u_{\alpha}}{\partial x_{\beta}}+\frac{\partial u_{\beta}}{\partial x_{\alpha}}\right)\left(\frac{\partial u_{\alpha}}{\partial x_{\beta}}+\frac{\partial u_{\beta}}{\partial x_{\alpha}}\right)}$

in which $\alpha$ and $\beta$ are the Cartesian indices.

\section{Solution algorithm}

The standard WCSPH suffers from pressure and density fluctuations ${ }^{25}$. Fatehi and Manzari ${ }^{26}$ showed that the velocity-pressure decoupling reduces the non-physical fluctuations, so a pseudo-constant density algorithm is introduced to remove the density and pressure fluctuations to improve the WCSPH. The algorithm has been implemented in $\mathrm{C}++$. To solve equations (9) to (12), a predictive corrective algorithm is applied as follows, with the discretization of the conservation of mass (Eq. 9):

$\frac{\rho_{i}^{n+1}-\rho_{i}^{n}}{\Delta t}=-\rho_{i}^{n+1}\left\langle\nabla \cdot V^{n+1}\right\rangle_{i}$

which leads to

$\rho_{i}^{n+1}=\frac{\rho_{i}^{n}}{1+\Delta t\left\langle\nabla \cdot V^{n+1}\right\rangle_{i}}$.

Dividing Eq. (10) by $\rho$ leads to:

$\frac{d \boldsymbol{V}}{d t}=\nabla \cdot\left(v_{p}\left(\nabla \boldsymbol{V}+\nabla \boldsymbol{V}^{\boldsymbol{T}}\right)\right)+\boldsymbol{g}-\frac{\nabla p}{\rho}$

where $v_{p}$ is $\mu_{p} / \rho$. The right-hand side terms of Eq. (17) are respectively acceleration terms due to viscous, buoyancy, and pressure forces. The SPH discretization of the above equation will be:

$\frac{\boldsymbol{V}_{i}^{n+1}-\boldsymbol{V}_{i}^{n}}{\Delta t}=\left(\boldsymbol{g}+\left\langle\nabla \cdot\left(v_{p}\left(\nabla \boldsymbol{V}+\nabla \boldsymbol{V}^{\boldsymbol{T}}\right)\right)\right\rangle_{i}-\left\langle\frac{\nabla p^{n}}{\rho}\right\rangle_{i}\right)$.

So, the velocity can be defined as:

$\boldsymbol{V}_{i}^{n+1}=\boldsymbol{V}_{i}^{*, n+1}-\Delta t\left\langle\frac{\nabla p^{n}}{\rho}\right\rangle_{i}$

where $\boldsymbol{V}_{i}^{*, n+1}$ is the intermediate velocity due to the buoyancy and viscous terms of fluid particle acceleration: 
$\boldsymbol{V}_{i}^{*, n+1}=\boldsymbol{V}_{i}^{n}+\left(\boldsymbol{g}+\widehat{\boldsymbol{B}}_{i}: \sum_{j} 2 \forall_{j} \boldsymbol{e}_{i j} \nabla W_{i j}\left(v_{p_{j}} \frac{\boldsymbol{V}_{j}^{n}-\boldsymbol{V}_{i}^{n}}{r_{i j}}-\boldsymbol{e}_{i j}\left\langle v_{p_{j}} \nabla \boldsymbol{V}\right\rangle_{i}^{n}\right)+\right.$

$\left.+\left(\nabla v_{p} \cdot \nabla \boldsymbol{V}^{T}\right)_{i}\right) \Delta t$.

Whereas $v_{p}$ is not constant, it is calculated by $v_{p_{i j}}=\mu_{p_{i j}} / \rho_{i j}$, where $\rho_{i j}$ is $\left(\rho_{i}+\rho_{j}\right) / 2$ and $\mu_{p_{i j}}$ is approximated by

$\mu_{p_{i j}}=\frac{2}{\frac{1}{\mu_{p_{i}}}+\frac{1}{\mu_{p_{j}}}}$

where $\mu_{p_{i}}$ and $\mu_{p_{j}}$ are calculated by using Eq. (13). Applying the divergence operator on the Eq. (19):

$\left\langle\nabla \cdot \boldsymbol{V}^{n+1}\right\rangle_{i}=\left\langle\nabla \cdot \boldsymbol{V}^{*, n+1}\right\rangle_{i}-\Delta t\left\langle\nabla \cdot \frac{\nabla p^{n}}{\rho}\right\rangle_{i}$

and substituting in Eq. (16) results in:

$$
\rho_{i}^{n+1}=\frac{\rho_{i}^{n}}{1+\Delta t\left(\left\langle\nabla \cdot \boldsymbol{V}^{*, n+1}\right\rangle_{i}-\Delta t\left\langle\nabla \cdot \frac{\nabla p}{\rho}\right\rangle_{i}^{n}\right)} \text {. }
$$

Then, the pressure at the next time-step according to Eq. (11) will be:

$p_{i}^{n+1}=p_{o}+c^{2}\left(\frac{\rho_{i}^{n}}{1+\Delta t\left(\left\langle\nabla \cdot \boldsymbol{V}^{*, n+1}\right\rangle_{i}-\Delta t\left\langle\nabla \cdot \frac{\nabla p}{\rho}\right\rangle_{i}^{n}\right)}-\rho_{0}\right)$.

As such, the velocity is corrected as follows:

$\boldsymbol{V}_{i}^{n+1}=\boldsymbol{V}_{i}^{*_{n+1}}-\left\langle\frac{\nabla p}{\rho}\right\rangle^{n+1} \Delta t$.

Lastly, the position vector for the particle $i$ is rearranged as follows:

$\boldsymbol{r}_{i}^{n+1}=\boldsymbol{r}_{i}^{n}+\boldsymbol{V}_{i}^{n+1} \Delta t$.

After fluid flow determination, the mass transport equation (concentration) is solved as follows:

$C_{i}^{n+1}=C_{i}^{n}+\left(\widehat{\boldsymbol{B}}_{i}: \sum_{j} 2 \alpha \forall_{j} \boldsymbol{e}_{i j} \nabla W_{i j}\left(\frac{C_{j}^{n}-C_{i}^{n}}{r_{i j}}-\boldsymbol{e}_{i j} \cdot\langle\nabla C\rangle_{i}^{n}\right)\right) \Delta t$.

The time step $\left(\Delta t=t^{n+1}-t^{n}\right)$ is calculated as

$\Delta t=\beta_{t} \min \left(\frac{\delta_{\min }}{V_{\max }}, \frac{\delta_{\min }^{2}}{v}, \sqrt{\frac{\delta_{\min }}{g}}, \frac{\delta_{\min }^{2}}{\alpha}\right)$

where $\beta_{t}$ is a constant coefficient between 0 and $1, \delta_{\text {min }}$ is the minimum distance between two neighbouring particles, and $V_{\max }$ is the maximum velocity magnitude of the particles.

The boundary conditions that dominate the wall boundaries are the no slip condition for velocity and Neumann boundary conditions for mass diffusion. The Neumann boundary condition for concentration $(C)$ is formulated for the wall boundary conditions as follows: $\frac{\partial C}{\partial n_{w}}=0$

where $n_{w}$ is the normal direction of the wall.

For the moving boundary condition, the pressure boundary condition is achieved as follows: $\left(\frac{\nabla p}{\rho}\right) \cdot \boldsymbol{n}_{w}=-\boldsymbol{a} \cdot \boldsymbol{n}_{w}+v \nabla^{2} \boldsymbol{V} \cdot \boldsymbol{n}_{w}+\boldsymbol{g} \cdot \boldsymbol{n}_{w}$

where $\boldsymbol{a}$ is the acceleration. If the body is fixed or moves with constant velocity or the normal vector of the surface is perpendicular to the acceleration vector (such as narrow blade rotation with constant angular velocity), this equation is simplified to the following form:

$\frac{\partial p}{\partial n_{w}}=0.0$.

The dummy particles theory of Lee et $a l .{ }^{25}$ has been found to be more compatible for the simulations presented herein. Two layers of dummy particles are arranged close to each wall boundary, similar to those particles defined by Lee et al. ${ }^{25}$. These particles have the same velocity as their corresponding wall particles. The dummy particles also have the same pressure and concentration as the wall in the normal direction in order to satisfy the Neumann boundary condition. Defects, tensile instability, and particle clustering are complications in SPH simulations. To avoid these unfavourable phenomena, a shifting algorithm similar to the particle shifting approach of $\mathrm{Xu}$ et al. ${ }^{27}$ was applied in the present study.

\section{Validation test}

The main problem involves two main physical aspects: mixing due to moving boundary and non-Newtonian power-law fluid flow. Hence, the results of the present algorithm are separately validated against previous results for each of the mentioned aspects.

\section{Moving boundary}

There are some Eulerian and grid-based results for the mixing due to moving boundaries. One of the studies which investigated the effect of rigid body motion on the fluid mixing was reported by Celik and Beskok ${ }^{28}$, who studied a channel-mixer consisting of a straight channel in which a circular cylinder oscillates transversely to improve the mixing. They considered a flat plate at the inlet flow (upstream of the cylinder) to remove the outlet vortices. The scheme of the model is shown in Figure 1. The cylinder motion is defined as follows:

$y=y_{\max } \sin \left(2 \pi f_{f} t\right)$

where $y_{\max }$ is equal to $0.4 D$ and $f_{f}$ is the forced frequency of the cylinder motion. The results of the present algorithm are compared with those of Celik and Beskok for the case:

$\mathrm{Re}=\frac{U D}{v}=100, \quad \frac{f_{f}}{f_{0}}=1.0, \quad \mathrm{Sc}=\frac{v}{\alpha}=1.0$

where $U$ is the average velocity acting on the cylinder and $f_{0}$ is the natural frequency of the vortex shedding. Approximately 20.000 particles are used for the SPH simulation. Contours of concentration and plot of variations of mixing index along the channel for the present simulations in comparison with the Celik and Beskok results are shown respectively in Figures 2 and 3. Mixing index is calculated as follows:

$D_{I}=\sqrt{\frac{1}{N} \sum_{j=1}^{N}\left(\frac{C_{j}-C_{\text {mean }}}{C_{\text {mean }}}\right)^{2}}$ 


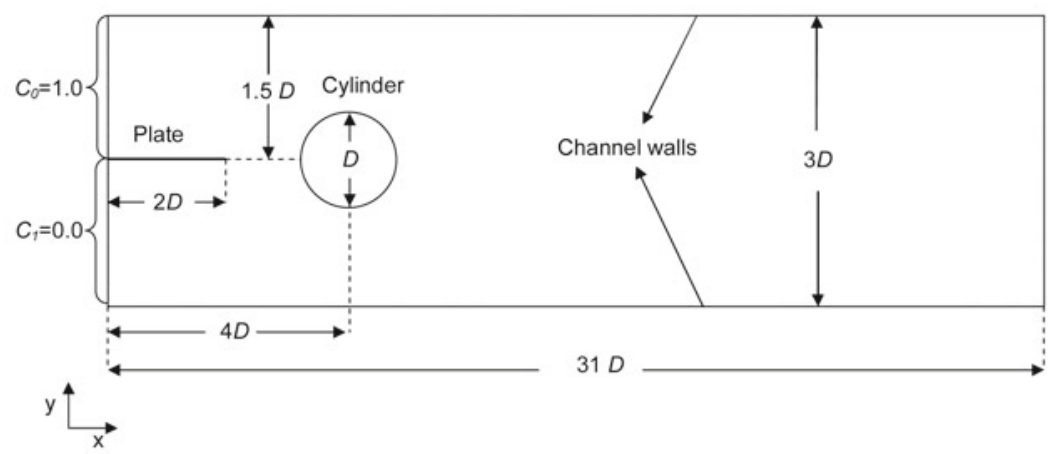

Figure 1. Schematic scheme of the channel-mixer involving a transversely oscillating cylinder to mix the entering fluids

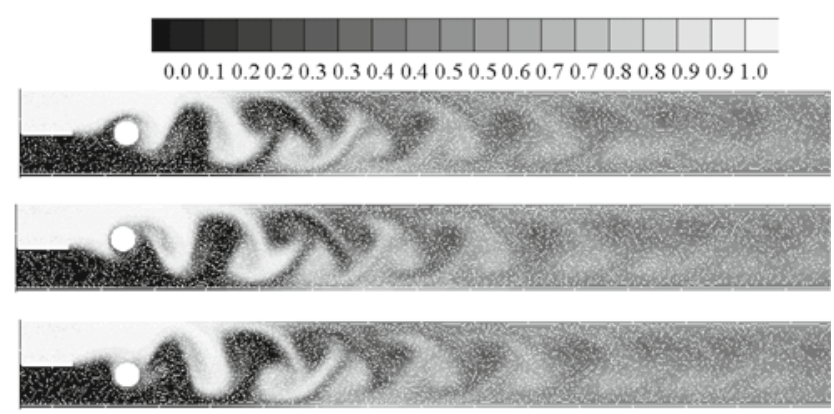

Figure 2. Concentration distribution along the channel involving the oscillating cylinder obtained from the present SPH simulation for the case: $\mathrm{Re}=100, \mathrm{Sc}=1.0, \mathrm{f}_{\mathrm{f}} / \mathrm{f}_{0}=1.0$

where $C_{\text {mean }}$ is the mean concentration at the initial time, $N$ is the number of particles, and $C_{j}$ is the local concentration indicated by the $j$-th particle concentration. When proper and homogeneous mixing occurs, the mixing index approaches zero.

As shown in Figure 3, there is good agreement between the results of the present simulation and those of Celik and Beskok ${ }^{28}$. For this case, the vortex shedding plays an important role in the mixing rate; the vortices create proper chaotic mixing and increase contact area among the particles. So, molecular diffusion is also enhanced. However, the Schmidt number for this case is very low $(\mathrm{Sc}=1.0)$, which is not applicable for the liquid mixing. It is expected that the low Reynolds number and mixing due to moving boundary in the closed chambers create the proper conditions for the mixing at high Schmidt numbers.

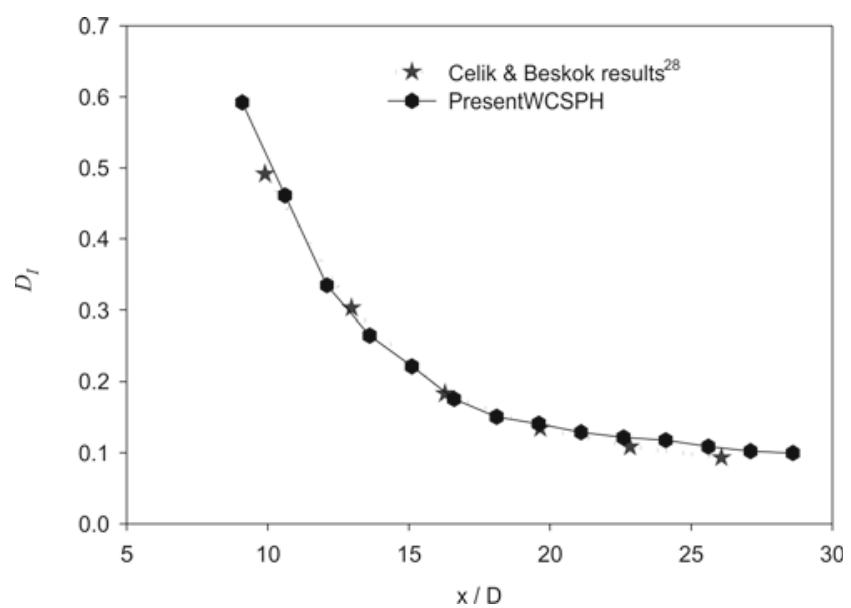

Figure 3. Variations of the mixing index along the channel for the present simulations in comparison with Celik and Beskok $^{28}$

\section{Non-Newtonian power-law verification}

To validate the method proposed for the non-Newtonian power-law modelling, the present code is tested for a traditional problem, which is the flow in the lid-driven cavity. The problem consists of a square cavity filled with an incompressible power-law fluid. The upper wall of the cavity moves with constant velocity. In Figure 4, the results of the present code are compared with those reported by Bell and Surana ${ }^{29}$ for lid-driven cavity flow. The Reynolds number of power-law fluid is calculated by

$\operatorname{Re}_{p l}=\frac{\rho U_{w}^{2-n_{p}} L_{w}^{n_{p}}}{k}$

where $U_{w}$ is the velocity of top lid, $L_{w}$ is the cavity length, and $n_{p}$ is power-law index. The results shown in Figure 4 have been achieved at $\operatorname{Re}_{\mathrm{pl}}=100$.
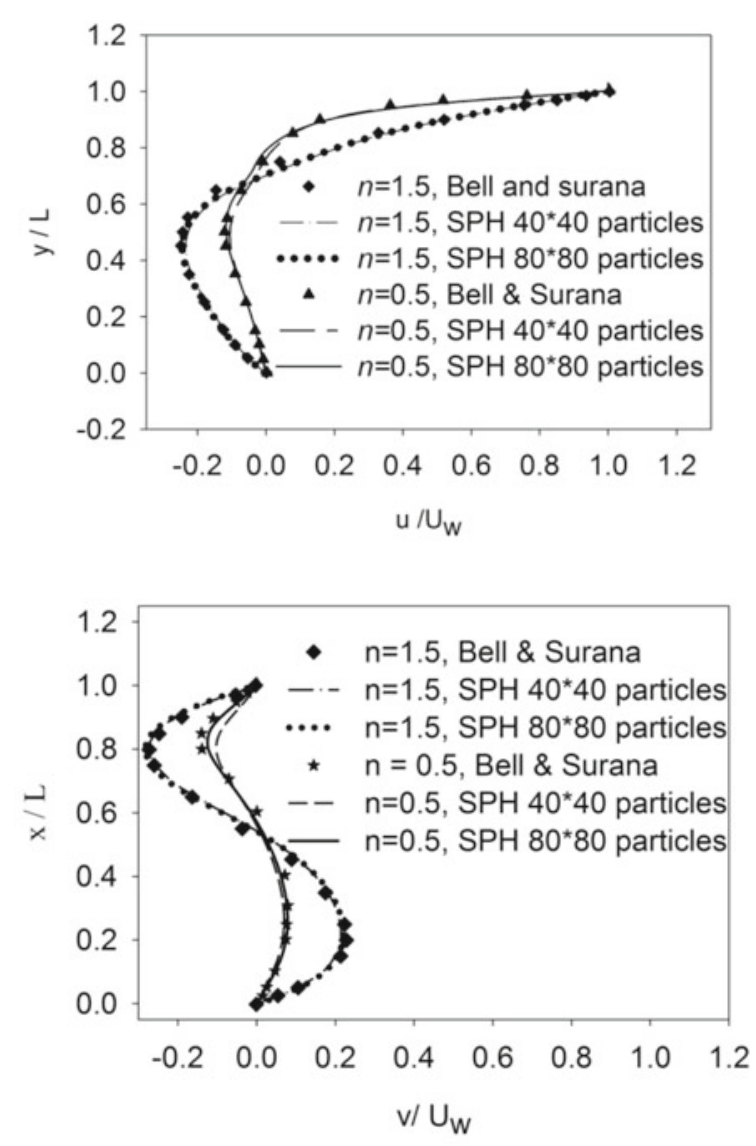

Figure 4. The Comparison of the vertical and horizontal middle sections velocity profiles of the lid-driven cavity between present study and Bell and Sourana ${ }^{29}$ results for $\mathrm{Re}_{\mathrm{pl}}=100$ at two values of power law index 


\section{RESULTS AND DISCUSSION}

\section{Problem Definition}

The main aim of the present study is the investigation of the fluid mixing in a two-blade mixer, which consists of a circular chamber in which two blades rotate to mix the fluids. The scheme of the mixer is shown in Figure 5. As shown in this figure, the two blades are perpendicular to each other within a long cylinder at the initial time, and when the system starts to work, the two blades rotate to mix the fluids. The fluids in the mixer are power-law fluids and have the same density but different concentration termed $C_{0}$ and $C_{1}$. In the present study, the effects of the power-law index, Reynolds number, and direction rotation of the blades on the fluid mixing are investigated.

As shown in Figure 5, it is assumed that the length of the mixer in comparison with its diameter is considerable. So, a two-dimensional model is considered for numerical modelling. For the mixer shown in Figure 5, $D / L$ is 2.4 where $L=0.00035 \mathrm{~m}$, and Schmidt number is constant and equals to:

$$
\mathrm{Sc}=\frac{\mathrm{Pe}}{\operatorname{Re}_{p l}}=\frac{\frac{L^{2} \omega}{2 \alpha}}{\frac{\rho\left(\frac{L \omega}{2}\right)^{2-n_{p}} L^{n}}{k}}=\frac{k}{\rho \alpha}\left(\frac{\omega}{2}\right)^{n_{p}-1}=1000
$$

where $\omega=\left|\omega_{1}\right|$ is the value of angular velocity of the blade. The effect of the number of particles has also been studied and the independent particle number has been considered for the simulation of the paddle mixer. About 6,200 particles are used for the present simulations.
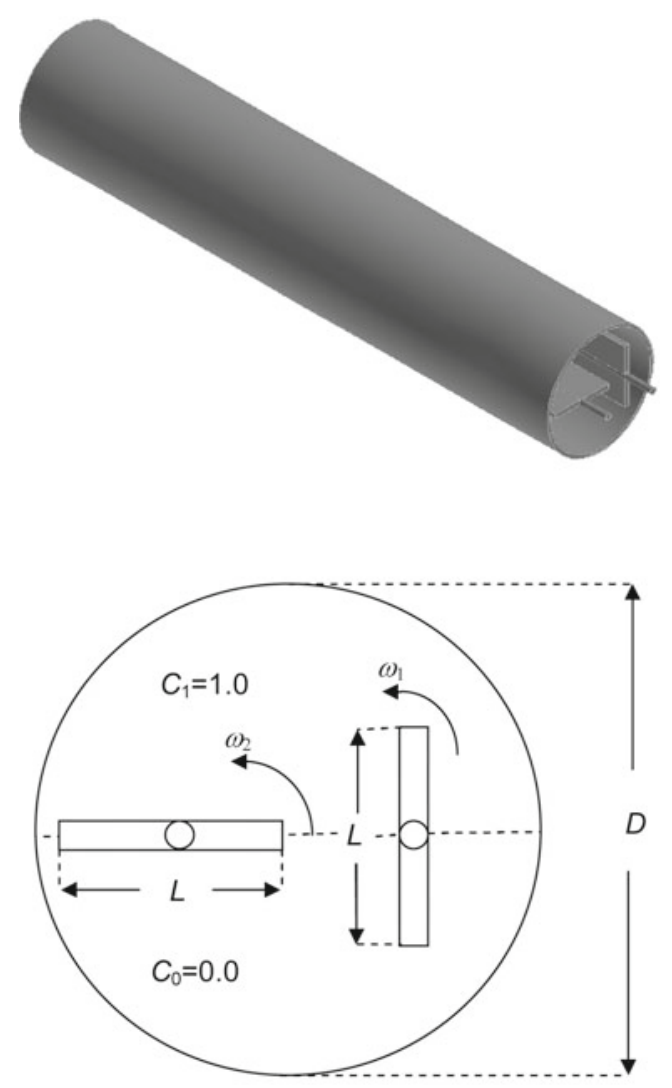

Figure 5. 3D schematic view and $2 \mathrm{D}$ scheme (cross section) of the two-blade mixer (initial condition)

\section{Mixing in the fully circular chamber}

The simplest chamber shape which can be considered for a two-blade mixer is a circular chamber. So, the initial step is to investigate the mixing behaviour of a two-blade circular mixer. Two power-law indices, $n_{p}=0.5$ and $n_{p}=1.1$, are considered and the mixing patterns and concentration distributions for different cycles of blade motion $\left(n_{c}\right)$ are shown in Figures 6 and 7. In these figures, the patterns of advection are shown in the left columns and the concentration distributions are shown in the right columns. For both of the power-law indices, there are regions at the top and bottom of the chamber in which the fluid particles move very slowly. In fact, the fluids which are at the top end and bottom end of the chamber are not in the main path of the advection mixing. Blade revolution forms distinct bands near the tip of the blade, and mixing strongly occurs in the regions where the bands are formed and stretched. Due to shear flow in the closed chamber, velocity of the fluid layers is different. So, the chance of contact among the particles increases. However, the region of well-mixed

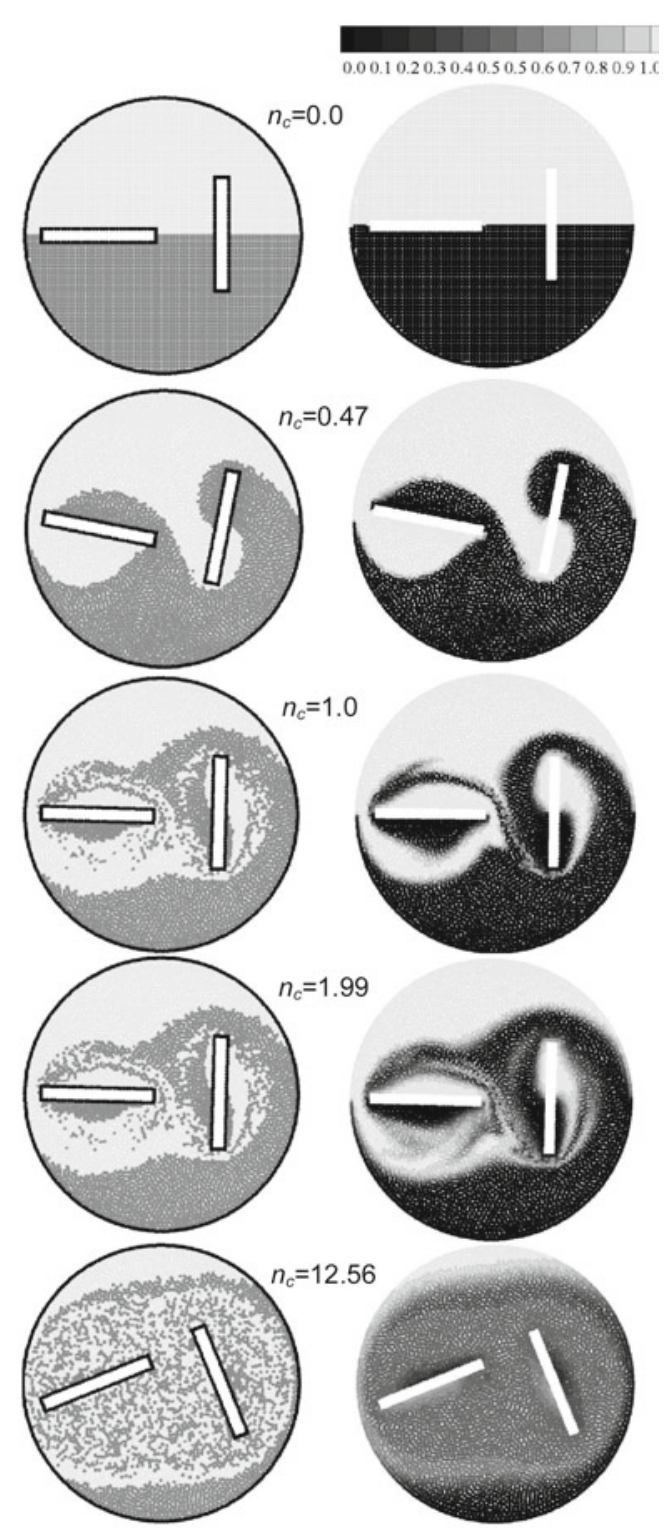

Figure 6. Patterns of the particles motion (first column) and concentration distribution (second column) for different revolution cycles for the case: $n_{p}=0.5, \omega_{1} / \omega_{2}=1$, $\mathrm{Re}_{\mathrm{pl}}=3.94, \mathrm{Sc}=1000$ 


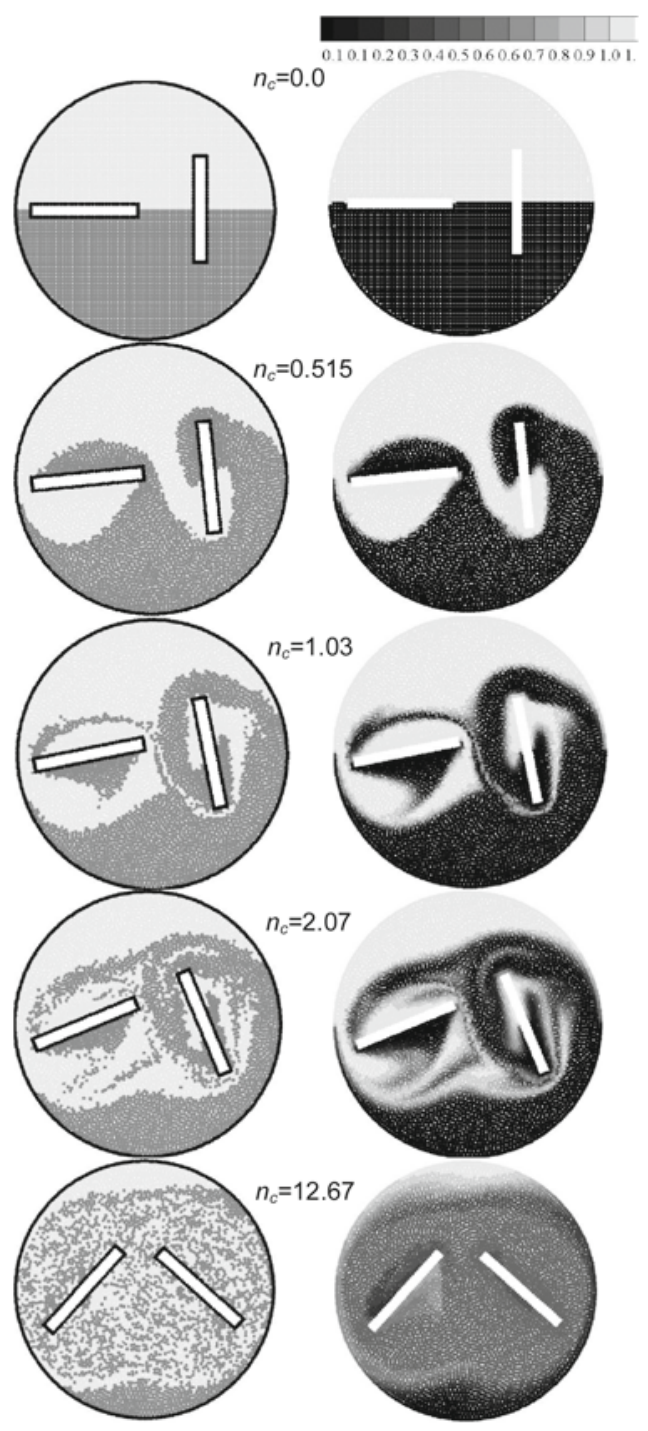

Figure 7. Patterns of the particles motion (first column) and concentration distribution (second column) for different revolution cycles for the case: $n_{p}=1.1, \omega_{1} / \omega_{2}=1$, $\mathrm{Re}_{\mathrm{pl}}=3.94, \mathrm{Sc}=1000$

fluids does not extended outside of the region where bands are formed; the particles which are outside of the blade sweep region remain largely unmixed, so efficient mixing does not occur. By tracing the mixing patterns, it is estimated that the modification of the geometry improves the mixing rate. The investigation of Figures 6 and 7 shows that, if the geometry is changed so that it is compatible with the mixing patterns, the mixing rate may improve. As such, the geometry is changed to a twin chamber mixer. It is necessary to mention that the areas of both the geometries are the same.

\section{Mixing in the twin circular chamber mixer}

As mentioned in the previous section, the geometry of the mixer is changed to remove the unmixed regions. As such, a twin chamber mixer, such as the one in Figure 8, is considered and examined. The results of SPH simulations for the new geometry for two power-law indices are shown in Figure 9. In this figure, the concentration distributions for the same condition as the fully circular geometry are shown. As expected, the unmixed regions have vanished. The standard deviation can be a proper indicator for the liquid mixers ${ }^{\mathbf{3 0}}$. It is also known as mixing index calculated by Eq. (34). To have a quantita-

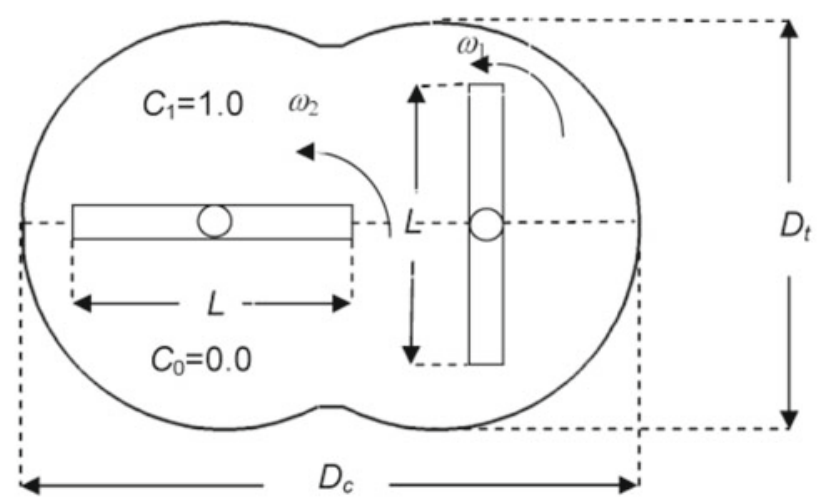

Figure 8. The scheme of the two-blade twin chamber mixer (initial condition)
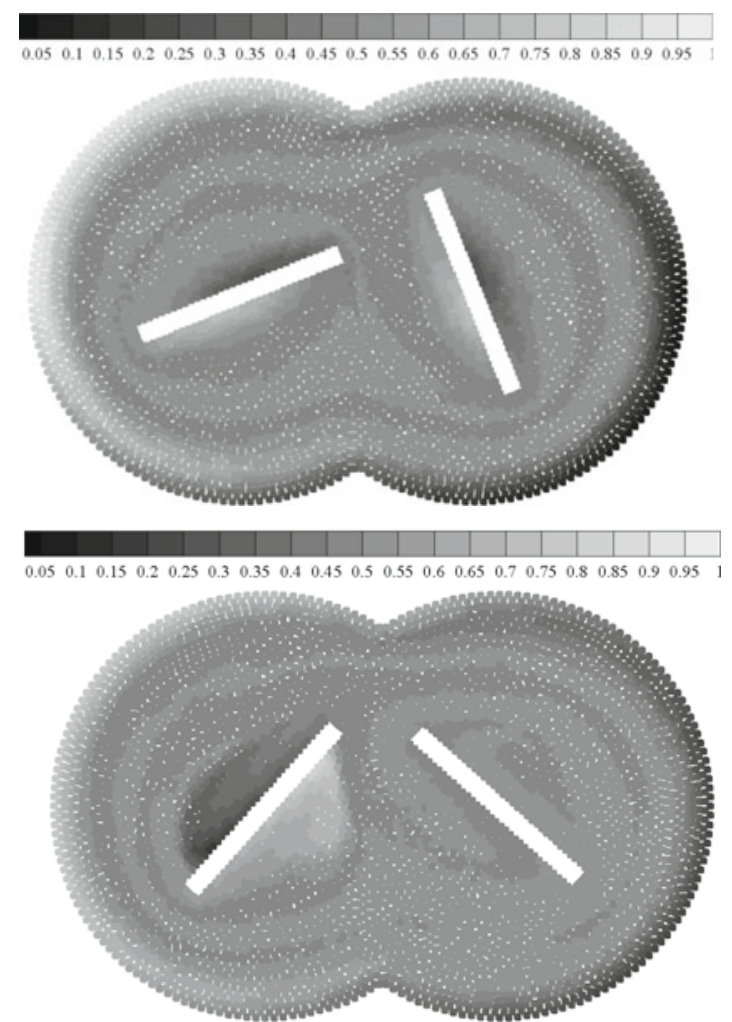

Figure 9. Concentration distributions for the case: $\mathrm{n}=0.5$, $\omega_{1} / \omega_{2}=1, n_{c}=12.56, \operatorname{Re}_{\mathrm{pl}}=3.93$ (left) and the case: $n_{p}=1.1, \omega_{1} / \omega_{2}=1, n_{c}=12.67, \mathrm{Re}_{\mathrm{pl}}=3.93$ (right)

tive analysis, mixing index versus the number of blade revolutions for both shapes is plotted in Figure 10. As shown, there is considerable improvement due to the

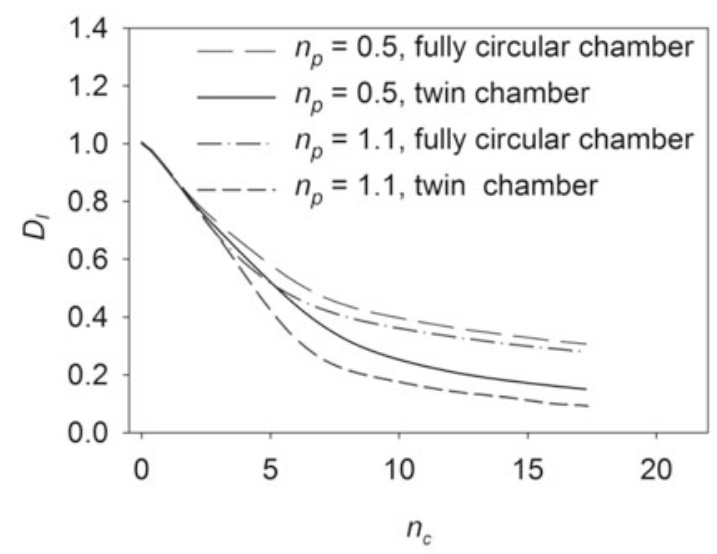

Figure 10. Variations of mixing index versus the number of blade revolutions for the fully circular chamber and twin chamber for two power-law indices 
geometry change; the mixing improvement for $n_{p}=1.1$ is about $63 \%$ and for $n_{p}=0.5$ is about $49 \%$. Due to geometry change, mixing improvement for the lower power-law index is less than that for the higher powerlaw index. As mentioned before, the shear flow plays a major role in the paddle mixers. There is a shear flow between the chamber wall and the blades. So, when the shear flow is weakened by the shear-thinning effect, the mixing rate decreases. In the shear flow, the fluid layers have a different velocity. So, in addition to the advection effects, the contacts among the particles are increased which causes an increase in the molecular diffusion. However, for the circular chamber, the shear flow does not effectively occur in the entire chamber.

\section{Effects of the power-law index on mixing}

As indicated in the plot of Figure 10, the effects of the geometry change on the case with $n_{p}=1.1$ are more than that with $n_{p}=0.5$. It was also mentioned that the effective shear flow has an important effect on the paddle mixers. The effect of shear flow is enhanced by increasing the power-law index in the twin chamber mixer. Hence, for three different Reynolds numbers and six power-law indices, the mixing index versus the number of blade revolutions is plotted in Figure 11. It is necessary to mention that the non-dimensional groups such as Reynolds number, Schmidt number, and the geometrical non-dimensional groups are assumed to be constant for each study. As shown in Figure 11, the mixing index regularly decreases by increasing the power-law index. These results confirm that the mixing process in the two-blade twin chamber mixer is more efficient for higher power-law indices. Although the three plots of Figure 11 are very similar, the times of

processes are different; by increasing Reynolds number, the mixing time decreases considerably.

It is necessary to mention that the case where the blades rotate at the same speed but in opposite directions was also examined. The results shown in Figure 12 also confirm that the mixing process is more efficient for the cases with higher power-law indices.

\section{Effects of the relative rotation direction of the blades on the mixing}

To investigate the effects of the rotation direction of the blades relative to each other on the mixing rate, the mixing index of two cases with different rotation direction of the blades are compared in Figure 13. In Figure 14, the stream lines for both cases (same rotation direction and opposite rotation direction of blades) for two power-law indices $\left(n_{p}=0.5\right.$ and $\left.n_{p}=1.1\right)$ are shown. By investigation of the stream lines and mixing paths, two mechanisms of mass transfer can be considered for the twin chamber mixers; mass transfer in each chamber of the mixer and mass transfer between the two chambers. The first mechanism is more efficient for the case with opposite rotation direction of the blades relative to each other $\left(w_{2} / w_{1}=-1\right)$. However, the second mechanism is stronger for the case with the same rotation direction of the blades $\left(w_{2} / w_{1}=1\right)$. The mixing paths of the first mechanism are significantly smaller than those of the second mechanism. Hence, as indicated in Figure 13, the mixing rate increases in the first cycles for the first mechanism in comparison with the second mechanism. However, maximum mixing occurs when the particles can travel through the entire mixer area. So, after a few cycles, the mixing index for the case with the same direction rotation of the blade reaches that of the case with the opposite direction rotation of the blade. Then,
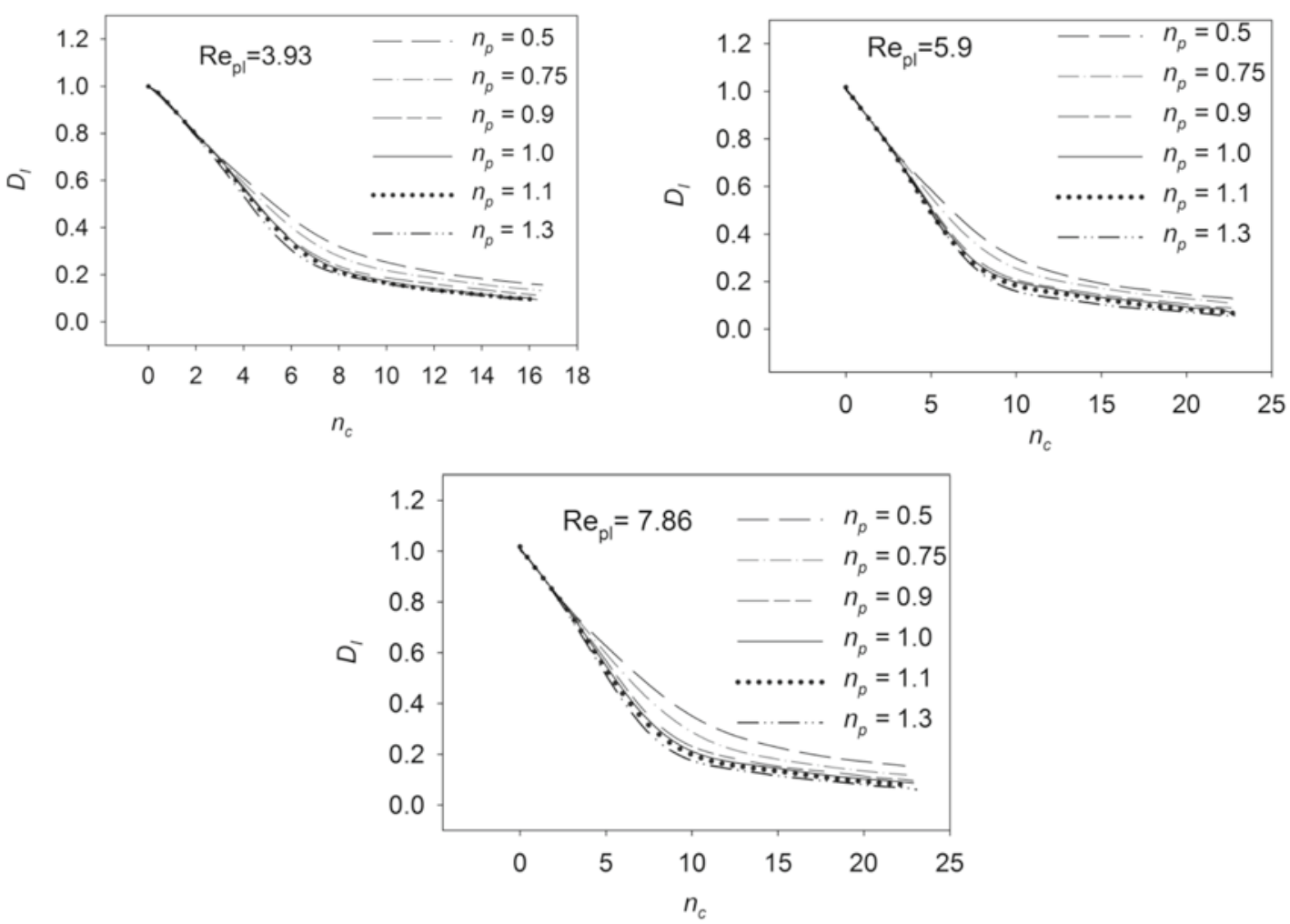

Figure 11. Variations of mixing index versus the number of blade revolutions for the twin chamber mixer for six power-law indices at three distinct Reynolds numbers for the case $\omega_{1} / \omega_{2}=1$ and $\mathrm{Sc}=1000$ 

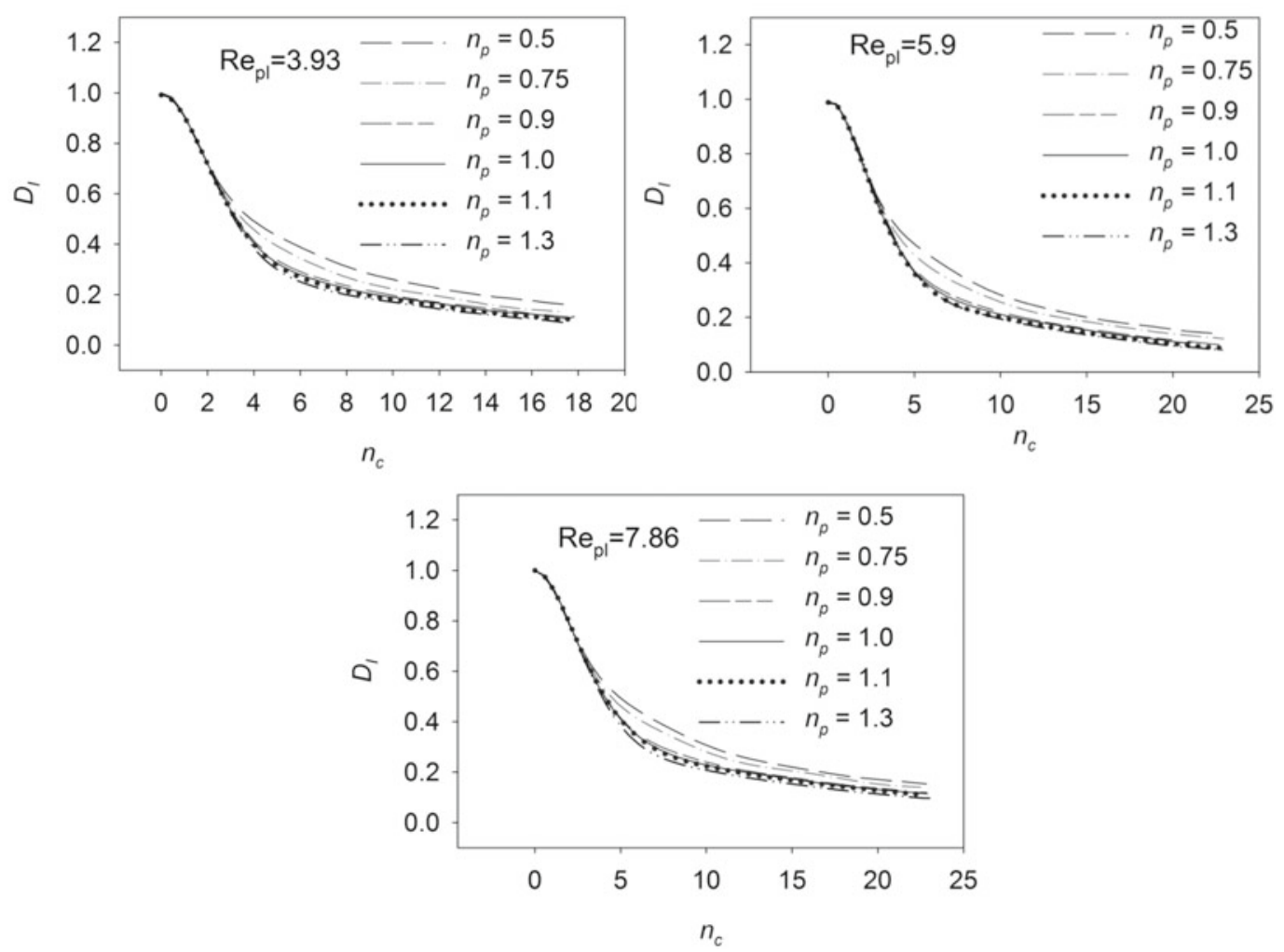

Figure 12. Variations of mixing index versus the number of blade revolutions for the twin chamber mixer for six power- law indices at three distinct Reynolds numbers for the case $\omega_{1} / \omega_{2}=-1$ and $\mathrm{Sc}=1000$

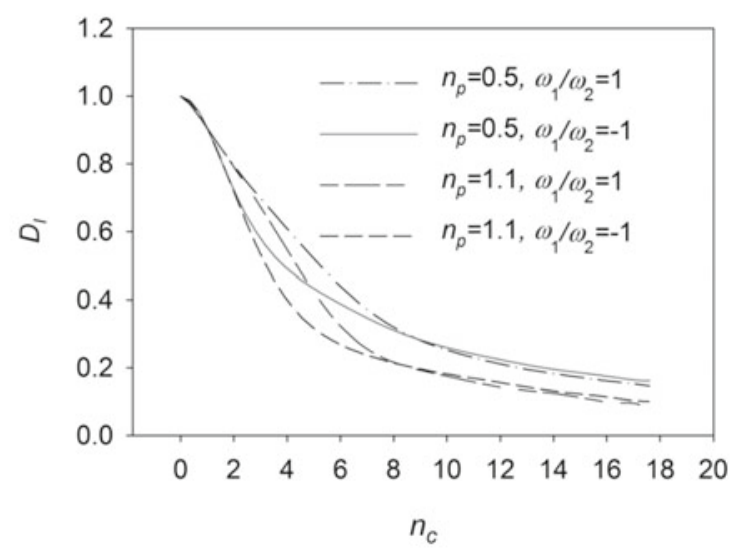

Figure 13. The comparison between the cases with same direction rotation of the blades $\left(\omega_{1} / \omega_{2}=1\right)$ and those with opposite direction rotation of the blades $\left(\omega_{1} / \omega_{2}=-1\right)$ for $\mathrm{Re}_{\mathrm{pl}}=3.93$

the mixing rate of the case with the same direction rotation of the blades increases slightly compared with that of the opposite rotation direction of the blades. 

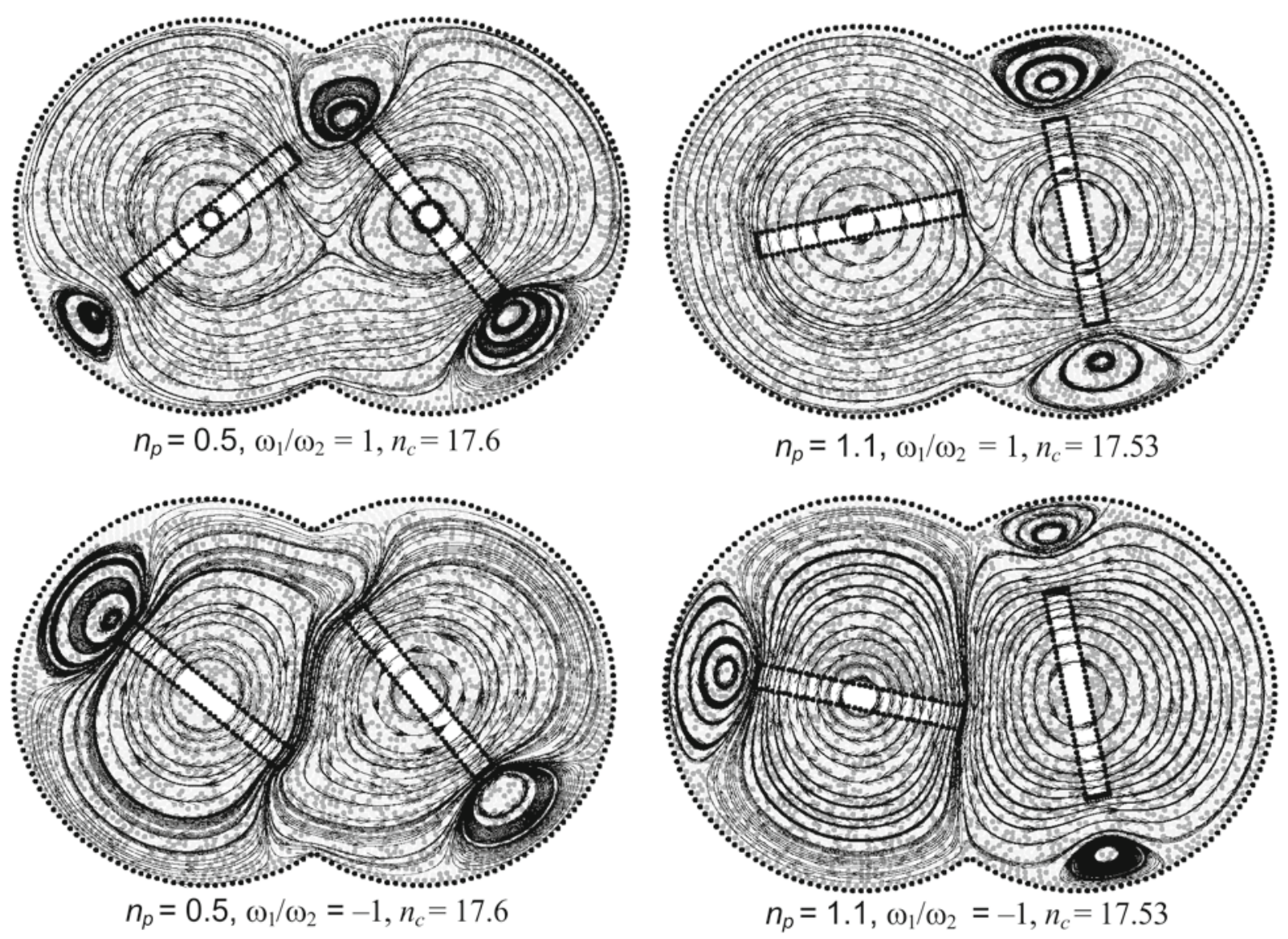

Figure 14. Streamlines for four different cases according to power-law index and rotation direction of the blades relative to each other for $\mathrm{Re}=3.93$

\section{CONCLUSION}

In the present study, a fully Lagrangian approach was applied to study the effects of the geometry and power-law index on the mixing rate of the miscible fluid in a two-blade paddle mixer. The results showed that for the fully circular chamber, there are regions at the top and bottom of the chamber in which the fluid particles move very slowly. In fact, the fluids which are at the top end and bottom end of the chamber are not in the main path of the advection mixing. So, the geometry was changed in a way that made it compatible with the patterns of particle motion.

The results showed that this change can decrease mixing index by at least $49 \%$. Also, the results showed that the twin chamber mixer is more efficient for the higher power-law indices. The effect of the rotation direction of the blades relative to each other is also investigated. The results show that opposite-direction rotating blades act faster than same-direction rotating blades. However, same-direction rotation is slightly more efficient at higher cycles of blade rotation.

\section{NOMENCLATURE}

$\begin{array}{ll}A & - \text { Area, }\left[\mathrm{m}^{2}\right] \\ \boldsymbol{a} & - \text { Acceleration, }\left[\mathrm{m} / \mathrm{s}^{2}\right] \\ \boldsymbol{B}, \widehat{B} & - \text { Corrective tensor for kernel gradients } \\ c & \text { - Speed of sound, }[\mathrm{m} / \mathrm{s}] \\ C & - \text { Concentration } \\ C_{\text {mean }} & \text { - Mean concentration at the initial time } \\ D, D_{\mathcal{c}}, D_{n} & - \text { Cylinder diameter, }[\mathrm{m}]\end{array}$

$e_{i j}$

F

$f$

$f_{0}$

$f_{f}$

g

h

k

L

$L_{w}$

$m$

$n$

N

$n_{p}$

$\boldsymbol{n}_{x}$

$$
\text { n }
$$$$
\begin{aligned}
& p \\
& r
\end{aligned}
$$$$
r
$$

r'

Re

$\mathrm{Re}_{\mathrm{pl}}$

Sc

$t$

$U_{w}$

$u$

$v$

V

$\forall{ }_{j}$

W

$y_{\text {max }}$
- The unit vector in the inter-particle direction (from $j$ to $i$ )

- Arbitrary tensor function

- Arbitrary scalar function

- Natural frequency of vortex shedding

- Forced frequency

- Buoyancy acceleration, $\left[\mathrm{m} / \mathrm{s}^{2}\right]$

- Smoothing length, [m]

- Consistency index, [Pa $\left.\mathrm{s}^{\mathrm{n}}\right]$

- Length of the stirrer, [m]

- Length of the cavity wall, [m]

- Mass, [kg]

- Time step counter

- Particles number

- Power-law index

- x-direction unit vector

- Normal vector of the surface

- Pressure, $[\mathrm{Pa}]$

- Position vector, [m]

- Sub integral variable, [m]

- Reynolds number

- Reynolds number defined for the power-law fluid

- Schmidt number

- Time, [s]

- Wall velocity, $[\mathrm{m} / \mathrm{s}]$

- Horizontal component of the velocity, $[\mathrm{m} / \mathrm{s}]$

- Vertical component of the velocity, $[\mathrm{m} / \mathrm{s}]$

- Velocity, [m/s]

- Volume of $j$-th particle, $\left[\mathrm{m}^{3}\right]$

- Weight or kernel function

- Amplitude of the cylinder oscillation, [m] 


\section{Greek symbols}

$\begin{array}{ll}\alpha & - \text { Mass diffusion coefficient, }\left[\mathrm{m}^{2} / \mathrm{s}\right] \\ \beta_{t} & - \text { Time-step coefficient } \\ \delta_{\min } & - \text { Minimum distance between two neighboring } \\ & \text { particles, [m] } \\ \varepsilon, & - \text { Displacement coefficient } \\ \mu & - \text { Viscosity, }[\mathrm{Pa} \mathrm{s}] \\ m_{\mathrm{p}} & - \text { Apparent viscosity for power-law fluid, [Pa s] } \\ v & - \text { Kinematic viscosity, }\left[\mathrm{m}^{2} / \mathrm{s}\right] \\ n_{p} & - \text { Apparent kinematic viscosity for power-law } \\ \dot{\gamma} & \quad \text { fluid, }\left[\mathrm{m}^{2} / \mathrm{s}\right] \\ \varrho, r_{0} & - \text { shear-rate, }[1 / \mathrm{s}] \\ \omega, w_{1}, w_{2} & - \text { Density, }\left[\mathrm{kg} / \mathrm{m}^{3}\right] \\ \forall & - \text { Angular velocity, }[1 / \mathrm{s}]\end{array}$

Subscripts

$\mathrm{i} \& \mathrm{j} \quad-$ Particle counter

$\alpha \& \beta \quad-$ Cartesian indices

\section{LITERATURE CITED}

1. Peryt-Stawiarska, S. \& Jaworski, Z. (2008). Fluctuations of the non-Newtonian fluid flow in a Kenics static mixer:An experimental study. Pol. J. Chem. Tech. 10(3), 35-37. DOI: 10.2478/v10026-008-0033-3.

2. Lai, A.C.K. \& Chen, F.Z. (2007). Comparison of a new Eulerian model with a modified Lagrangian approach for particle distribution and deposition indoors. Atmos. Environ. 41(25), 5249-5256. DOI: 10.1016/j.atmosenv.2006.05.088.

3. Zhang, X. \& Ahmadi, G. (2005). Eulerian-Lagrangian simulations of liquid-gas-solid flows in three-phase slurry reactors. Chem. Eng. Sci. 60(18), 5089-5104. DOI: 10.1016/j. ces.2005.04.033.

4. Lenaerts, T. \& Dutré, P. (2009). Mixing fluids and granular materials. Comput. Graph. Forum 28(2), 213-218. DOI: 10.1111/j.1467-8659.2009.01360.x.

5. Robinson, M., Cleary, P. \& Monaghan, J.J. (2008). Analysis of mixing in a twin cam mixer using Smoothed Particle Hydrodynamics. AIChE 54(8), 1987-1998. DOI: 10.1002/aic.11530.

6. Orthmann, J. \& Kolb, A. (2012). Temporal blending for adaptive SPH. Comput. Graph. Forum 31(8), 1-12. DOI: 10.1111/j.1467-8659.2012.03186.x.

7. Gingold, R.A. \& Monaghan, J.J. (1977). Smoothed Particle Hydrodynamics: theory and application to non-spherical stars. Mon. Not. Roy. Astron. Soc. 181, 375-389.

8. Lucy, L.B. (1997). A numerical approach to the testing of the fission hypothesis. Astron. J. 82, 1013-1024. DOI: $10.1086 / 112164$.

9. Antoci, C., Gallati, M. \& Sibilla, S. (2007). Numerical simulation of fluid-structure interaction by SPH. Comput. Struct. 85(11-14), 879-890. DOI: 10.1016/j.compstruc.2007.01.002.

10. Potapov, S., Maurel, B., Combescure, A. \& Fabis, J. (2009). Modeling accidental-type fluid-structure interaction problems with the SPH method. Comput. Struct. 87(11-12), 721-734. DOI: 10.1016/j.compstruc.2008.09.009.

11. Eghtesad, A., Shafiei, A.R. \& Mahzoon, M. (2011). A new fluid-solid interface algorithm for simulating fluid structure problems in FGM plates. J. Fluid Struct. 30, 141-158. DOI: 10.1016/j.jfluidstructs.2012.02.005.

12. Hosseini, S.M. \& Amanifard, N. (2007). Presenting a modified SPH algorithm for numerical studies of fluid-structure interaction problems. IJE Transactions B: Applications 20(2), 167-178.

13. Shamsoddini, R., Sefid., M. \& Fatehi, R. (2014). ISPH modelling and analysis of fluid mixing in a microchannel with an oscillating or a rotating stirrer. Eng. Appl. Comp. Fluid. 8(2), 289-298.
14. Cheremisinoff, N.P. (2002). Handbook of water and wastewater treatment technology. UK: Butterworth Heinemann.

15. Youcefi, A., Anne-Archard, D., Boisson, H.C. \& Sengelin, M. (1997). On the influence of liquid elasticity on mixing in a vessel agitated by a two-bladed impeller. J. Fluid Eng-T. ASME, 119(3), 616-622. DOI: 10.1115/1.2819289.

16. Han, Y., Wang, J.J., Gu, X.P. \& Feng, L.F. (2012). Numerical simulation on micromixing of viscous fluids in a stirred-tank reactor. Chem. Eng. Sci. 74, 9-17. DOI: 10.1016/j. ces.2012.02.018.

17. Bohl, D. (2007). Experimental investigation of the fluid motion in a cylinder driven by a flat plate impeller. J. Fluid Eng-T. ASME 129(1), 737-746. DOI: 10.1115/1.2734186.

18. Bohl, D., Mehta, A., Santitissadeekorn, N. \& Bollt, E. (2011). Characterization of mixing in a simple paddle mixer using experimentally derived velocity fields. J. Fluid Eng-T. ASME 133(6), 061202-061210. DOI: 10.1115/1.4004086.

19. Kor, Y.K., Prince, R.G.H. \& Fletcher, D.F. (2008). Using CFD to identify means of reducing power consumption for mixing and suspension in paper pulp stock chests. Asia-Pac. J. Chem. Eng. 3(2), 144-150. DOI: 10.1002/apj.126.

20. Paul, E.L., Atiemo-Obeng, V. \& Kresta, S.M. (2004). Handbook of industrial mixing: science and practice. USA: Wiley-Interscience.

21. Wendland, H. (1995). Piecewise polynomial, positive definite and compactly supported radial functions of minimal degree. Adv. Comput. Math. 4(1), 389-396. DOI: 10.1007/ BF02123482.

22. Dehnen, W. \& Aly, H. (2012). Improving convergence in smoothed particle hydrodynamics simulations without pairing instability. Mon. Not. Roy. Astron. Soc. 425(2), 1068-1082. DOI: 10.1111/j.1365-2966.2012.21439.x.

23. Bonet, J. \& Lok, T.S. (1999). Variational and momentum preservation aspects of Smooth Particle Hydrodynamic formulation. Comput. Meth. Appl. Mech. Eng. 180(1-2), 97-115. DOI: 10.1016/S0045-7825(99)00051-1.

24. Fatehi, R. \& Manzari, M.T. (2011). Error estimation in Smoothed Particle Hydrodynamics and a new scheme for second derivatives. Comput. Math. Appl. 61(2), 482-498. DOI: 10.1016/j.camwa.2010.11.028.

25. Lee, E.S., Moulinec, C., Xu, R., Laurence, D. \& Stansby, P. (2008). Comparisons of weakly compressible and truly incompressible algorithms for the SPH mesh free particle method. J. Comput. Phys. 227(18), 8417-8436. DOI: 10.1016/j. jcp.2008.06.005.

26. Fatehi, R. \& Manzari, M.T. (2011). A remedy for numerical oscillations in weakly compressible Smoothed Particle Hydrodynamics. Int. J. Numer. Meth. Fl. 67(9), 1100-1114. DOI: $10.1002 /$ fld.2406.

27. Xu, R., Stansby, P. \& Laurence, D. (2009). Accuracy and stability in incompressible SPH (ISPH) based on the projection method and a new approach. J. Comput. Phys. 228(18), 6703-6725. DOI: 10.1016/j.jcp.2009.05.032.

28. Celik, B. \& Beskok, A. (2009). Mixing induced by a transversely oscillating circular cylinder in a straight channel. Phys. Fluids 21, 0736011-0736019.

29. Bell, B. \& Surana, K. (1994). P-version least squares finite element formulation for two-dimensional, incompressible, non-Newtonian isothermal and non- isothermal fluid flow. Int. J. Numer. Meth. Fl. 18, 127-162. DOI: 10.1002/fld.1650180202.

30. Pianko-Oprych, P. \& Jaworski, Z. (2009). CFD modelling of two-phase liquid-liquid flow in a SMX static mixer. Pol. $J$. Chem. Tech. 11( 3), 41-49. DOI: 10.2478/v10026-009-0034-x. 